treatment effect is typically 2 years. Only small changes can be assessed over this period, normally about 1-2 mSASSS units. It is hard to define that this is clinically relevant. The most important for treatment is to show that there is inhibition of structural progression in comparison to untreated patients, especially as axial $\mathrm{SpA}$ is a lifelong disease and 1 unit over 30 years still leads to severe ankylosis of the spine.

Disclosure of Interest: None declared

DOI: 10.1136/annrheumdis-2018-eular.7656

\section{SP0044 WHAT DO WE LEARN FROM RCTS ON THE TREATMENT EFFECT ON STRUCTURAL PROGRESSION IN AXSPA?}

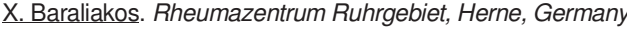

The introduction of tumour necrosis factor inhibitors (TNFis) about 20 years ago has led to the hope of disease modification of ankylosing spondylitis, since biologics showed for the first time a decrease of inflammatory activity on MRI, with the latter being theoretically also directly linked to new bone formation. However, the first open-label extensions of randomized-controlled trials with a treatment duration of 2 years failed to show any positive effect on the radiographic progression in AS patients when compared to historical cohorts that had not been exposed to biologics. Nevertheless, later data indicated that this lack of influence on radiographic progression might have been due to many different reasons that were not taken into account in these first analyses, such as the radiographic status of patients at baseline, CRP levels or insufficient duration of follow-up. Furthermore, most recent data from MRI studies also indicated that the most important link to influence radiographic progression with biologics might not be the suppression of inflammation but the protection of bone to show tissue metaplasia to post-inflammatory findings, while early suppression of inflammation might be the key to even completely inhibit radiographic progression in AS patients.

Indeed, most recent cohort data have been able to demonstrate an association between TNF-blocker treatment and reduced risk of spinal structural progression (e.g. formation of syndesmophytes). Furthermore, early escalation of treatment from NSAIDs to biologics and long-term treatment with biologics have also independently been able to show positive effects on radiographic progression in patients with AS. Finally, also newer biologics such as IL-17A inhibitors have also provided promising results in terms of overall low radiographic progression rates as measured by validated scoring systems.

Currently, first head-to-head trials of different biologics are underway to examine any possible differences between the available compounds with a primary outcome of their effect on spinal radiographic progression.

It remains to be shown whether and how these results will also become clinically relevant in terms of decrease or even inhibition of spinal mobility restrictions, in order to be able to postulate a 'real' disease modifying effect of biologic treatment in axial spondyloarthritis.

Disclosure of Interest: None declared

DOI: 10.1136/annrheumdis-2018-eular.7840

\section{THURSDAY, 14 JUNE 2018}

\section{Reproductive issues in rheumatology}

\section{SP0045 OESTROGENS, IMMUNE RESPONSE AND AUTOIMMUNE DISEASES}

M. Cutolo, on behalf of Eular Study Group on Neuroendocrinelmmunology of the Rheumatic Diseases. Research Lab. Division Rheumatology. Dept Internal Medicine University of Genova Italy, genova, Italy

Sex hormones are implicated in the immune response, with estrogens as enhancers at least of the humoral immunity and androgens and progesterone (and glucocorticoids) as natural immune-suppressors. Several physiological, pathological and therapeutic conditions may change the serum oestrogen milieu including the menstrual cycle, pregnancy, postpartum period, menopause, elderly, chronic stress, altered circadian rhythms, inflammatory cytokines, use of glucocorticoids, oral contraceptives, and steroid hormonal replacements. Cortisol and melatonin circadian rhythms are altered, at least in rheumatoid arthritis (RA), and partially involve also sex hormone circadian synthesis and levels. Abnormal regulation of aromatase activity (i.e. increased activity) by inflammatory cytokines production (i,e, TNF-alpha, IL-1, IL-6) may partially explain the abnormalities of peripheral oestrogen synthesis in RA (i.e. increased availability of 17-beta estradiol and possible metabolites in synovial fluids) and in systemic lupus srythematosus (SLE). In the synovial fluids of RA patients the increased oestrogen concentration are observed in both sexes and are more specifically characterised by the hydroxylated forms, in particular 16alpha-hydroxyestrone, that is a mitogenic and cell proliferative endogenous hormone. Local effects of sex hormones in autoimmune rheumatic diseases seems to consist mainly in modulation of cell proliferation.

Epidemiological evidence indicates that during the fertile age women are more often affected by rheumatic diseases than men, particularly autoimmune diseases. As a matter of fact, rheumatic disorders with autoimmune involvement such as RA or SLE, result from the combination of several predisposing factors, that include the relationships between epitopes of the trigger agent (i.e. virus), the status of the stress response system including the hypothalamic-pituitary-adrenocortical axis (HPA) and mainly the effects of the gonadal hormones (hypothalamic-pituitary-gonadal axis - HPG)

The pre-or post-menopausal serum sex hormonal status is a further factor influencing the rate of rheumatic diseases. It is therefore important, whenever possible, to evaluate epidemiologic data broken down into age (for example 10 year age band) and sex-specific group before making inferences. Obviously, sex hormones seem to play an important role as modulators of both disease onset and perpetuation and show circadian rhythms together with cortisol.

Sex hormones are implicated in the immune response, with estrogens as enhancers at least of the humoral immunity and androgens and progesterone (and glucocorticoids) as natural immune-suppressors. Low concentrations of gonadal and adrenal androgens [testosterone ( $\mathrm{T})$ /dihydrotestosterone (DHT), dehydroepiandrosterone (DHEA) and its sulphate (DHEAS), respectively] levels, as well as reduced androgens/estrogens ratio, have been detected in serum and body fluids (i.e. blood, synovial fluid (SF), smears, salivary) of male and female RA patients, as well as in SLE, supporting the possible pathogenic role for the decreased levels of the immune-suppressive androgens. However, respect to serum levels of estrogens, interestingly they are not significantly changed which is in strict contrast to androgen levels in RA patients (reduced).

As a matter of fact, sex hormones can exert also local actions (paracrine) in the tis sues in which they are formed or enter the circulation and both $T$ and 17-beta estradiol seem to exert dose and time-dependent effects on cell growth and apoptosis. These effects, as well as important influences on gene promoter of Th1/Th2 cytokines and the recently discovered increased SF oestrogen concentrations might suggest new interesting roles for estrogens at least in RA. Finally estrogens exert important epigenetic actions on cell proliferation. Estrogens act as key fac tors in cellular proliferation and differentiation as well as cancer development and progression (prostate). The expression of oestrogen receptor (ER)- $\beta$ appears to be lost during prostate cancer progression through hypermethylation mechanism Epigenetic drugs such as 5-aza-2'-deoxycytidine (5-AZAC) and Trichostatin A (TSA) showed efficacy in restoring ER $\beta$ expression in prostate cancer cells These observations highlights that the strategy of merging epigenetic and hormonal therapies might be beneficial also in inflammatory/autoimmune diseases (synovial tissue)

\section{REFERENCES}

[1] Cutolo M, et al. Estrogen's effects in chronic autoimmune/inflammatory dis eases and progression to cancer. Expert Rev Clin Immunol 2014;10:31-9.

[2] Cutolo M, et al. Ann. Rheum. Dis 2005;64:212-6.

[3] Cutolo M, et al. Autoimmun. Rev 2005;4:497-502.

[4] Cutolo M, et al. Ann. N. Y. Acad. Sci 2005;1051:372-8.

[5] Janele D, et al. Ann. N. Y. Acad. Sci 2006;1069:168-82.

[6] Motawi TK, et al. Life Sci 2018 Feb 15;pii: S0024-3205(18)30069-9

Disclosure of Interest: None declared

DOI: 10.1136/annrheumdis-2018-eular.7725

\section{SP0046 \\ WHAT WE NEED TO CONSIDER IN PHYSICIAN-PATIENT COMMUNICATION ON SEXUAL PROBLEMS IN DIFFERENT RHEUMATIC CONDITIONS}

M. Ostensen. Department of Rheumatology, St.Olavs Hospital, Kristiansand, Norway

Quality of life (QOL) is often reduced in patients with chronic diseases. Sexual activity and enjoyment constitute an important aspect of QOL. Sexuality is a neglected area of QOL in patients with rheumatic disease.

Sexual problems among patients are common and often increase with disease duration. Both disease related factors and the psychological response to chronic disease can impair sexual functioning. General disease symptoms like pain, fatigue, disease activity, and impaired physical function contribute to reduced sexual activity in both genders. However, psychological factors like depression, anxiety, negative body image and low self-esteem play an important role. Sexua dysfunction can create frustration and distress, and if chronic increase anxiety and depression, and damage interpersonal relationships. 
Sexual health is rarely addressed by health professionals (HP), and it is as a rule not spontaneously reported by patients. HPs may feel awkward to intrude into the intimate sphere of a patient, and patients may feel ashamed of revealing their sexual dysfunction.

A first step to improve the physician-patient communication in this area is to include sexuality in the curriculum of health professionals teaching how to address and evaluate sexual function. Assessment of sexual function by validated instruments includes both frequency of intercourse as well as sexual desire, arousal, orgasm, and sexual satisfaction. In women vaginal lubrication and in men erectile function and ejaculation are part of the evaluation. Assessment of disease activity and comorbidities helps to detect physical components of sexual dysfunction.

Barriers regarding communication on sexual activity should be identified and overcome. Assessment of sexual function may be assigned to a specially trained nurse, an occupational therapist or a psychologist of the interdisciplinary team. Referral to specialists in urology, gynaecology or sexual medicine may also help patients to get a better sex life. A major point for restoring sexual and reproductive health in patients of both genders is to achieve optimal disease control.

\section{REFERENCE:}

[1] Østensen M. Sexual and reproductive health in rheumatic disease. Nat Rev Rheumatol 2017;13(8):485-493.

Disclosure of Interest: None declared DOI: 10.1136/annrheumdis-2018-eular.7832

\section{SP0047 CHALLENGES IN THE MANAGEMENT OF DIFFERENT RHEUMATOLOGIC DISORDERS DURING PREGNANCY: LESSONS FROM THE REGISTRIES}

R. Fischer-Betz. Rheumatology, Heinrich-Heine-University, Duesseldorf, Germany

Registers have considerably expanded our knowledge in many fields of rheumatology. In particular, the Biologics Registers contribute to an enormous increase in knowledge on the "real world" safety of the rapidly growing treatment options. Family planning in the case of RD is a particular challenge in the physician-patient relationship and requires an optimal strategy. The impact of pregnancy on the underlying disease or the impact of maternal disease on the outcome of pregnancy is not yet fully understood. In addition there is still a high unmet need of data on drug safety as women with wish to have children or pregnant women are excluded from registration studies for ethical reasons. Therefore, systematic and prospective observation in daily care is the best possibility to collect data on this subject. Data options range from clinical-based cohort studies (e.g. the PROMISSE study), prospective pregnancy exposure studies (e.g. the MotherToBaby studies) to national birth registries. Recently, pregnancy registers in women with $\mathrm{RD}$ have been established in several European countries. Together with other studies, these registers will hopefully add to improved expertise in the future Disclosure of Interest: R. Fischer-Betz Grant/research support from: GSK, UCB, Consultant for: Medac, UCB, Speakers bureau: Abbvie, Chugai, Janssen, Lilly, UCB, Pfizer

DOI: 10.1136/annrheumdis-2018-eular.7770

\section{THURSDAY, 14 JUNE 2018}

\section{Clinical challenges in giant cell arteritis in 2018}

\section{SP0048 A CASE OF PULSELESS STROKE}

F. Muratore. Rheumatology Unit, Azienda USL-IRCCS di Reggio Emilia, Reggio Emilia, Italy

Giant cell arteritis (GCA) is the most common form of vasculitis in individuals aged 50 years and over. GCA typically affects large and medium-sized arteries, with a predilection for the extracranial branches of the carotid artery. Patients with GCA usually present with symptoms and signs that are directly related to the artery that is affected, with or without constitutional manifestations. The most dreaded complication of GCA is visual loss, which affects about one in six patients and is typically caused by arteritis of the ophthalmic branches of the internal carotid artery. Before the advent of glucocorticoid treatment, the prevalence of visual complications was high. Increasing awareness by physicians of the symptoms of GCA and advances in diagnostic techniques over the past twenty years have also contributed to a substantial decline in the frequency of permanent visual loss. Ischaemic brain lesions are less common than visual lesions, and mostly result from vasculitis of the extradural vertebral or carotid arteries. In the case of both the eye and the brain, ischaemic damage is thought to result from arterial stenosis or occlusion that occurs secondary to the inflammatory process.
A patients with giant cell arteritis with extensive extra and intracranial large vessel involvement effectively treated with cyclophosphamide followed by mycophenolate mofetil will be presented. Diagnosis and management of ischaemic complications in giant cell arteritis will be discussed.

Disclosure of Interest: None declared

DOI: 10.1136/annrheumdis-2018-eular.7763

\section{SP0049 HEAD GAMES WITH GCA AND GCS}

M. Matza. Rheumatology, Massachusetts General Hospital, Boston, USA

Case 1: This is a $65 \mathrm{y} / \mathrm{o}$ woman with osteoporosis, depression and recently diagnosed polymyalgia rheumatica characterised by shoulder and hip girdle pain and stiffness with elevated inflammatory markers. She developed recurrent symptoms and new-onset jaw and tongue claudication in the setting of a prednisone taper. She was found to have a bulging right temporal artery and biopsy confirmed giant cell arteritis. She was placed on prednisone $60 \mathrm{mg}$ daily with resolution of her symptoms, but developed worsening symptoms of depression and anxiety with insomnia on high dose prednisone. She subsequently attempted suicide by intentional medication overdose and carbon monoxide poisoning. She was admitted to the psychiatry ward and additionally found to have a varicella zoster skin rash. Upon resolution of the zoster infection she was started on tocilizumab by subcutaneous injection weekly and prednisone was successfully tapered over 4.5 months without recurrence of symptoms.

Case 2: This is a $63 \mathrm{y} / 0$ man with ITP on monthly rituximab and chronic prednisone $10 \mathrm{mg}$ daily, hypertension, hyperlipidemia and osteoarthritis who developed worsening shoulder and neck pain for two months with more recent onset of scalp tenderness and left-sided vision changes for two weeks. He was found to have left optic neuropathy, elevated inflammatory markers, and an MRI demonstrating enhancement of the left temporal artery. Left temporal artery biopsy was normal. He was given pulse dose intravenous methylprednisolone for suspected giant cell arteritis and transitioned to high dose oral prednisone with improvement in muscu loskeletal symptoms, inflammatory markers and stabilisation of his vision. He subsequently developed insomnia, hyperactivity and talkativeness and was diagnosed with steroid-induced mania, which improved with antipsychotics and benzodiazepines as per the psychiatry service. He was discharged home and after three days developed new vision loss of the right eye on prednisone $60 \mathrm{mg}$ daily. On exam, he was found to have progressive visual field loss of the left eye and new inferior visual field loss of the right eye with disc oedema of the right optic nerve. Right temporal artery biopsy was negative. He was again given pulse dose intravenous methylprednisolone followed by oral methylprednisolone and ultimately received tocilizumab intravenously prior to discharge.

Disclosure of Interest: None declared

DOI: 10.1136/annrheumdis-2018-eular.7655

\section{THURSDAY, 14 JUNE 2018}

\section{Do we still need biopsies to diagnose Sjögren's and autoimmune myostitis?}

\section{SP0050 OPPORTUNITIES AND CHALLENGES OF IMAGING IN PRIMARY SJÖGREN'S}

\section{S. Jousse-Joulin. Rheumatology, Cavale Blanche Hospital, Brest, France}

Sjogren's syndrome (SS) is a chronic autoimmune inflammatory disorder of exocrine glands. Its diagnosis relies solely on a combination of clinical and laboratory findings. However, recent developments have shown that imaging techniques may have additional value in detecting salivary glands abnormalities in pSS. In general, sialography is considered to be the most reliable of the imaging methods. Salivary gland scintigraphy is very sensitive and especially useful in early stages of the disease. Nevertheless, both imaging techniques are used by only minority of rheumatologists for diagnosis of pSS because of the invasive character of sialography and the low specificity of scintigraphy. MRI has shown a good sensitivity and specificity to detect structural abnormalities in pSS but few centres have access to the specific know-how. A recent development is the increased interest in ultrasonography (US) as a tool to assess major salivary glands. Ultrasonography of the salivary glands (SGUS) appears to be an inexpensive commonly available noninvasive technique that does not cause complications and inconvenience to the patient, although the data are somewhat conflicting. Although, the recent but preliminary American classification criteria for Sjögren syndrome do not include salivary gland such imaging technique ${ }^{1}$ this procedure has clearly demonstrated high impact on classification of Sjögren patients ${ }^{2-5}$ and demonstrated good diagnostic properties. ${ }^{5}$ A systematic literature review ${ }^{6}$ has shown a paucity of data regarding the metric properties of ultrasound, making interpretation and 